\title{
Parking in the City
}

\author{
P. $\check{\mathrm{SEBA}}{ }^{a, b, c}$ \\ ${ }^{a}$ University of Hradec Králové, Hradec Králové, Czech Republic \\ ${ }^{b}$ Institute of Physics, Academy of Sciences of the Czech Republic \\ Prague, Czech Republic \\ ${ }^{c}$ Doppler Institute for Mathematical Physics and Applied Mathematics \\ Faculty of Nuclear Sciences and Physical Engineering, Czech Technical University \\ Prague, Czech Republic
}

We show that the spacing distribution between parked cars can be obtained as a solution of certain linear distributional fixed point equation. The results are compared with the data measured on the streets of Hradec Králové. We also discuss a relation of these results to the random matrix theory.

PACS numbers: 05.20.--y, 02.50.Cw, 82.20.Mj

\section{Introduction}

Our aim is to describe the spacing distribution between cars parked parallel to the curb somewhere in the center of a city. We will assume that the street is long enough to enable a parallel parking of many cars. Moreover we assume that there are no driveways or side streets in the segment of interest and that the street is free of any kind of marked parking lots or park meters. So the drivers are not biased to park at some particular positions. On the contrary: they are free to park the car anywhere provided they find and empty space to do it. In addition we assume that there are no cars parked permanently on the street (i.e. we assume that the majority of the cars leaves the street during the night).

The standard way to describe such random parking is the continuous version of the random sequential adsorption model known also as the "random car parking problem" - see $[1,2]$ for review. It is a well-studied process where the cars are parked without overlapping onto randomly chosen positions. All cars have the same length $l_{0}$ and all parking attempts are regarded as independent. Let us assume that the street has a length $L \gg l_{0}$. The particular parking attempt goes as follows: choose a random position $x$ on the street. If the interval $\left(x-l_{0} / 2, x+l_{0} / 2\right)$ 
is free, park the car with the middle at $x$. If this interval is not free choose another random position $x$ and try again.

Finally a jamming limit is reached and no additional cars can be parked. In the limit $L \rightarrow \infty$ it has been proved by Renyi [3] that the jamming limit is achieved when there are $N=q L / l_{0}$ cars parked with $q=0.74759 \ldots$ The mean distance $\bar{D}$ between the cars is in this case equal to $\bar{D}=l_{0}(1-q) / q$, i.e. $D \approx 0.3376 \ldots l_{0}$.

There is a considerable interest to optimize the parking problem. First of all it has been made out that as many as $30 \%$ of all cars going around in the city center are cruising for parking [4] and hence contribute substantially to the traffic congestion. The attempts to organize somehow the city parking by introducing parking fees and similar methods lead often to ambiguous results - see $[5,6]$. Mainly because the city traffic, that include together the cruising and parked cars, represents a nonlinear system that is difficult to optimize. It is therefore of interest to understand the mechanism that finally leads to a self-organized parking distribution in cases when all regulations are absent.

\section{Theory}

We focus on the distribution of the bumper to bumper distances $D$ between the parked cars. The first attempt (known to us) to collect such empirical data has been done by Rawal and Rodgers [7] in the center of London. The results were surprising since they differed substantially from the spacing distribution predicted by the random car parking model. The gap distribution for various versions of the random sequential adsorption model has been studied in detail in $[8,9]$ since it has direct consequences among others for the distribution of cracks in brittle materials [10]. In the most simple continuous case (random car parking model) the spacing distribution $P(D)$ behaves like

$$
P(D) \approx 0.4217[1-2 \ln (D)-(1-D)(1.379-0.365 D)]
$$

for small $D$. Therefore $P(D) \rightarrow \infty$ for $D \rightarrow 0$ [11]. But the results from London demonstrated clearly that in reality $P(D) \rightarrow 0$ as $D \rightarrow 0$. In addition Abul-Magd [12] pointed out that the spacing between parked cars in London is well described by the particle spacing distribution of a one-dimensional gas of interacting particles in thermal equilibrium (Dyson gas) [13] and hence by the random matrix theory [14].

There is a fundamental difference between the approach of Rawal and Rodgers [7] and that of Abul-Magd [12]. Rawal and Rodgers used a car parking model based on random adsorption. This approach in its simplest version does not assume any particular interaction between cars. But it is irreversible and does not lead to an equilibrium state. The approach of Abul-Magd on the contrary works with an equilibrium distribution. But it anticipates certain car interaction (repulsive logarithmic potential) and the resulting equilibrium state has a (magic) pre-set temperature. 
Novel measurements showed that the results presented by Rawal and Rodgers are not representative enough. We measured similar data on various streets in the center of Hradec Králové (Czech Republic). It comes out that the spacing distribution between the parked cars is not universal and depends on the particular traffic situation. The same phenomenon has been observed also in Ann Arbor (USA) [15] where it was demonstrated that the "temperature" of the Dyson gas depends on the street where the cars park.

Our aim is to explain these observations. We use a simplified reversible version of the random car parking model without anticipating any particular car interaction or driver behavior. We show that the model leads to results that are in amazing agreement with the observed data.

The description of the model is very simple. Let us recall that in the random car parking model all cars have the same length $l_{0}$ and are parked on a street of length $L, L \gg l_{0}$.

Let $X_{i}$ denote the position of the center of the $i$-th car and let the cars be always counted starting with the left end of the street: $X_{(i+1)}>X_{i}$. So if a car parks or leaves the street the car positions $X_{i}$ have to be relabeled.

The model works with repeated discrete time steps and is formulated as follows: We start with two cars parked at the ends of the street: $X_{1}=0, X_{2}=L$. These two cars will not move during the iterative model steps. They just mark the street ends simplifying in such a way the model formulation. All remaining cars participate in the model iterations. Each step of the model consists of two parts:

- Part 1. A car arrives to the street and tries to park there. The length of the parking slots $D_{k}$ is obtained as $D_{k}=X_{k+1}-X_{k}-l_{0}$. However a car of a length $l_{0}$ can use a slot $D_{k}$ only if $D_{k}>l_{0}$. If such slots are available the arriving car takes out randomly one of them and parks there. In doing so the driver parks to a preferred position. We assume that this parking preference is the same for all drivers and describe it as follows: Let $k_{0}$ be the index referring to the chosen parking lot. Then the driver parks the car to a position

$$
X=X_{k_{0}}+l_{0}+a\left(D_{k_{0}}-l_{0}\right),
$$

where $a \in(0,1)$ is random number with a probability density $P(a)$. For $a=0$ the car parks immediately in front of the car delimiting the parking lot from the left without leaving any empty space (very unworthy way to park). For $a=1 / 2$ it parks directly to the center of the lot and for $a=1$ it stops exactly behind the car on the right.

If there is not a vacant parking lot the part 1 of the model is skipped.

- Part 2. Each parked car leaves the street with a probability $p$.

If there are no other cars than those at the positions $X=0$ and $X=L$ this step is skipped. 
On a street of the length $L$ one can crowd maximally $N_{\max }=L / l_{0}$ cars. It is clear that probability $p$ determines the actual number $N$ of the parked cars. Since for $N p>1$ on the average more than one car leave the street in one step, the maximal number of parked cars fluctuates around the number $N=1 / p$ provided $N<N_{\max }$. Here we are mainly interested in the situation when all possible parking lots are taken, i.e. $N \approx N_{\max }$. So we will discuss the model for the parameter $p \leq 1 / L$.

The probability density $P(a)$ reflects the parking volatility of the drivers. For instance when $P(a)=\delta(a-0.5)$ all drivers park orderly punctually to the center of the lot.

In what follows we will assume:

- A symmetric probability distribution of the parking volatility $P(a)=$ $P(1-a)$.

- All parking lots are occupied. So a car can park only when another car leaves the street.

- The model is in an equilibrium stage so the spacing distribution does not change with time.

- To simplify the description we neglect events when more than one car leave the street within one step.

Let us focus on a randomly chosen triple of cars parked one after another and denote their positions as $X_{n-1}, X_{n}, X_{n+1}$. They define two spacings $D_{1}=$ $X_{n}-X_{n-1}-l_{0}$ and $D_{2}=X_{n+1}-X_{n}-l_{0}$. Let us assume now that the car $X_{n}$ leaves the street. The two spacings $D_{1}, D_{2}$ merge into one interval $D$ :

$$
D=D_{1}+D_{2}+l_{0} \text {. }
$$

When another car arrives it parks to the lot $D$ according to the rule (2) and splits it into two new spacings $\tilde{D}_{1}, \tilde{D}_{2}$ :

$$
\tilde{D}_{1}=a\left(D-l_{0}\right), \quad \tilde{D}_{2}=(1-a)\left(D-l_{0}\right) .
$$

Inserting (3) to (4) leads finally to

$$
\tilde{D}_{1}=a\left(D_{1}+D_{2}\right), \quad \tilde{D}_{2}=(1-a)\left(D_{1}+D_{2}\right) .
$$

Due to the symmetry of $P(a)$ the variables $a$ and $1-a$ have identical probability distributions. Moreover: there is nothing special on the chosen triple of cars. In the course of time the car $X_{n+1}$ leaves as well and is replaced by another one. This breaks the statistical dependence of $a$ and $1-a$ and we write finally

$$
\tilde{D}_{1} \triangleq a\left(D_{1}+D_{2}\right), \quad \tilde{D}_{2} \triangleq b\left(D_{1}+D_{2}\right),
$$

where $a$ and $b$ are independent random numbers with identical probability density. The variables $D_{1}, D_{2}, \tilde{D}_{1}, \tilde{D}_{2}$ are regarded as statistically independent copies of the car spacings and the symbol $\triangleq$ means that left and right sides of (6) have the same distribution. Distributional fixed point equations of this type are well studied see for instance $[16,17]$. It can be solved by iterations. Inserting the expression 
for $\tilde{D}_{2}$ into the equation for $\tilde{D}_{1}$ we obtain $\tilde{D}_{1} \triangleq a_{1}\left(D_{1}+a_{2}\left(D_{1}+a_{3}\left(D_{1}+\ldots\right)\right)\right)$ which leads to

$$
\tilde{D}_{1} \triangleq a_{1}+a_{1} a_{2}+a_{1} a_{2} a_{3}+a_{1} a_{2} a_{3} a_{4}+\ldots
$$

where $a_{1}, a_{2}, \ldots$ are all statistically independent with identical distribution $P(a)$. The same equation holds of course also for $\tilde{D}_{2}$.

The relation (7) can be easily rewritten as [18]

$$
\tilde{D} \triangleq a(D+1) \text {. }
$$

Let us note that the relation (6) does not depend on $l_{0}$. Hence the resulting spacing distribution is identical for all car lengths.

\section{Results and discussion}

When $a$ is an independent and identically distributed (i.i.d.) variable in $[0,1]$ (the drivers park everywhere inside the parking lot with equal probability) the solution of Eqs. (7), (8) is known and given by the Dickman distribution. It is well known from the number theory and describes the decomposition of large integers into prime factors [19]. The Dickman distribution is plotted in Fig. 1. The result is of interest since it points out that there is a direct link between the spacing distribution of the parked cars and the abstract number theory, quite in similarity with the nowadays intensively studied link between the distribution of prime numbers and the random matrix theory. In addition it shows that in the case of i.i.d. variable $a$ the spacing probability density $\rho(D)$ is constant: $\rho(D) \approx 0.5614 \ldots$ for $D<1$.

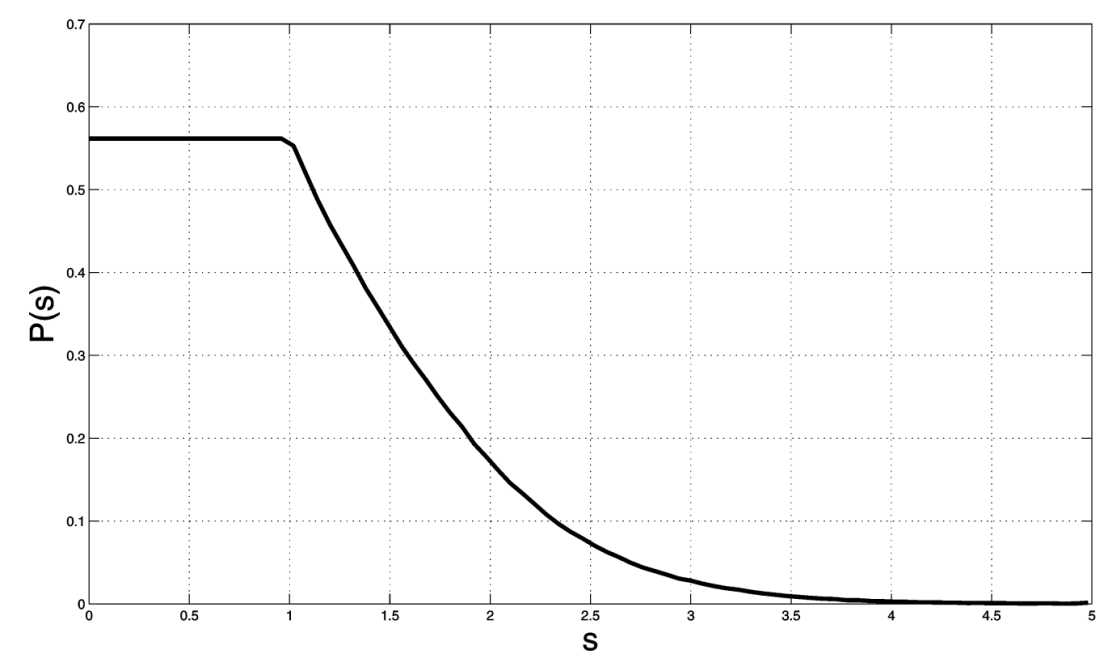

Fig. 1. The plot of the Dickman distribution.

Certainly: a parking behavior with $a$ being i.i.d. is unrealistic one because it fully ignores the parking maneuver. However the appearance of the Dickman 
distribution is inspiring. It shows among others that there is a relation between Eq. (8) and certain differential equations (let us note that the Dickman function is usually defined as a solution of the Dickman differential equation). Since it is generally more simple to work with differential equation than with the abstract relation (8) we will use this example and elucidate how the differential equation comes into the game. Following [18] and denoting by $\rho(D)$ the probability density of the distance $D$ we define the cumulative distribution function $R(t)$ of $D$ :

$$
R(t):=\operatorname{prob}[D \leq t]=\int_{0}^{t} \rho(s) \mathrm{d} s
$$

Using (8) we have

$$
R(t)=\int_{0}^{1} \operatorname{prob}[a(D+1) \leq t] P(a) \mathrm{d} a=\int_{0}^{1} R\left(\frac{t}{a}-1\right) P(a) \mathrm{d} a .
$$

Changing the variables: $s=t / a-1$ we obtain an integral equation for $R(t)$ :

$$
R(t)=t \int_{t-1}^{\infty} R(s) P\left(\frac{t}{s+1}\right) \frac{\mathrm{d} s}{(s+1)^{2}} .
$$

For the i.i.d. variable $a$ we have $P(a)=1$ and Eq. (11) simplifies to

$$
R(t)=t \int_{t-1}^{\infty} R(s) \frac{\mathrm{d} s}{(s+1)^{2}}
$$

It leads - by differentiating both sides with respect to $t$ and a simple per parts integration - directly to the Dickman equation

$$
t \rho^{\prime}(t)+\rho(t-1)=0 .
$$

As already mentioned the condition $P(a)=1$ is unrealistic. More likely is a situation with $P(0)=P(1)=0$. One candidate is the $\beta(2,2)$ distribution $P(a)=6 a(1-a)$. Inserting it into (11) we find that in this case the spacing probability density $\rho$ is a solution of

$$
t^{3} \frac{\mathrm{d}^{2}}{\mathrm{~d} t^{2}}\left(\frac{\rho(t)}{t}\right)=6 \rho(t-1)
$$

The solution is not analytic and can be obtained stepwise. For $t \in[0,1]$ we have $\rho(t-1)=0$ (there are no negative spacings) and hence $\rho(t)=c_{1} t+c_{2} t^{2}$ with $c_{1}, c_{2}$ being some constants. Using this solution for $\rho(t-1)$ we obtain for $t \in[1,2]$ :

$$
\rho(t)=3\left(c_{2}-c_{1}\right)+3\left(2 c_{2}-c_{1}\right) t \ln (t)+6 c_{2} t^{2}(\ln (t)-1)+c_{3} t^{2}+c_{4} t,
$$

where the new constants $c_{3}, c_{4}$ have to be chosen such that $\rho(t)$ and $\rho^{\prime}(t)$ are continuous at $t=1$ :

$$
c_{3}=3 c_{1}-8 c_{2}, \quad c_{4}=c_{1}+12 c_{2} .
$$

Using this expression we can obtain a solution $t \in[2,3]$ and so on (it becomes kind of cumbersome and we will not write it here). So the solution is fully determined by $c_{1}, c_{2}$. They have to be chosen in such a way that the density $\rho$ is properly normalized 


$$
\int_{0}^{\infty} \rho(t) \mathrm{d} t=\int_{0}^{\infty} t \rho(t) \mathrm{d} t=1
$$

Surprisingly enough — the density $\rho$ obtained as a solution of (8) for $P(a)=$ $6 a(1-a)$ and hence of $(14)$ is practically undistinguishable from the level spacing distribution obtained for a Gaussian orthogonal ensemble (GOE) of random matrices - see Fig. 2. In principle one can obtain a differential equation also for $P(a)=\beta(3,3)$ and so on. However the calculation becomes readily difficult. So it is more suitable to abandon the differential equation approach and to work directly with the relation (8) and solve it by iterations. And again: for $P(a)=\beta(3.3,3.3)$ the distribution $\rho$ coincides in admirable way with the prediction obtained for the Gaussian unitary ensemble (GUE). It has to be stressed that we used the proper GUE result for the comparison and not its approximation by the Wigner surmise.

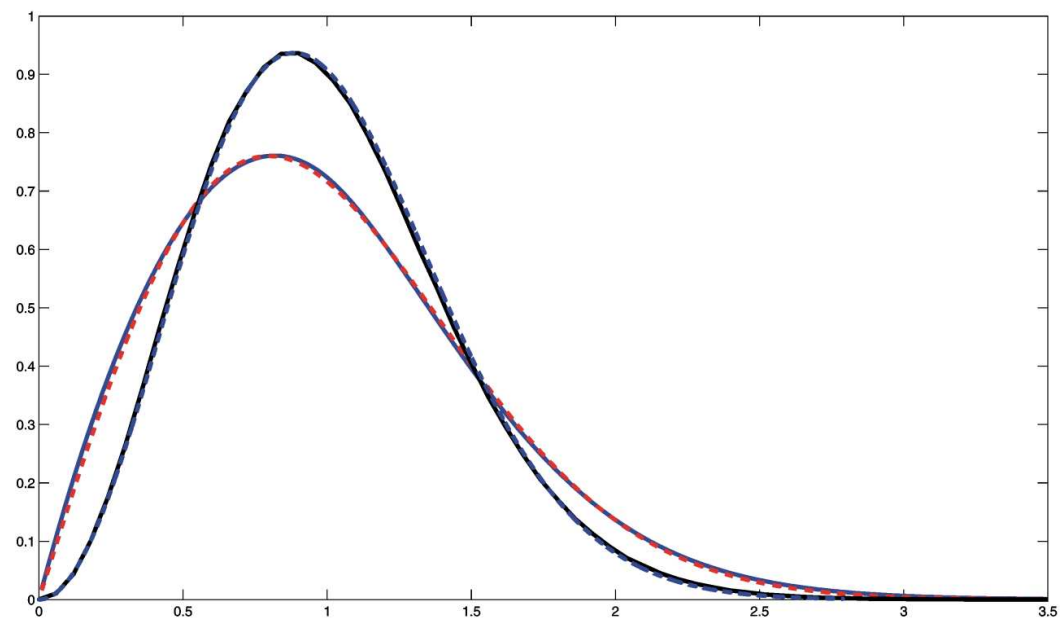

Fig. 2. The solution of (14) (full line) is compared with the level spacing distribution for GOE. Similarly for $P(a)=\beta(3.3,3.3)$ the solution of (8) is compared with the prediction of GUE.

These results demonstrate that there could be a link between the relation (8) and the random matrix theory. Moreover the relation (8) goes far beyond the parking problem. It describes a general split-and-merge process as well as a series of other systems ranging from Poisson-Dirichlet processes, GEM distribution, genom recombination and more — see for instance [20-24].

Let us return back to the parked cars. To obtain some "experimental" data we measured the distance between the cars parked on two different streets in the center of Hradec Králové (Czech Republic). One of them was a standard street with two-way traffic and cars parked on the right side (let us note that we drive on the right side of the street in Czech Republic). The second one was a one-way 
street and the cars were parked on its left side. In both cases we measured 1200 car spacings. The point is that the parking maneuver is a bit different in both cases. It is more simple to park a car on the left side, since the driving wheel is left as well and one has - so to say - a better sight. In other words: the probability density $P(a)$ depends where we park. The left side parking allows more tricky maneuvering and hence the usage of smaller lots. (In the Czech Republic it is not a habit to enlarge the lot by pushing cars with bumper that is known as a custom practice for instance in France.) The resulting distribution displays indeed an influence of this fact.

There are two possibilities how to simulate the parking situation: We can use the two steps of the model and iterate them for a time long enough to reach the steady situation or use directly the relation (8). Both approaches lead finally to the same result. Hence the events when two and more cars leave the street in one step do not influence the final spacing distribution. (We neglected them when deriving (8).)

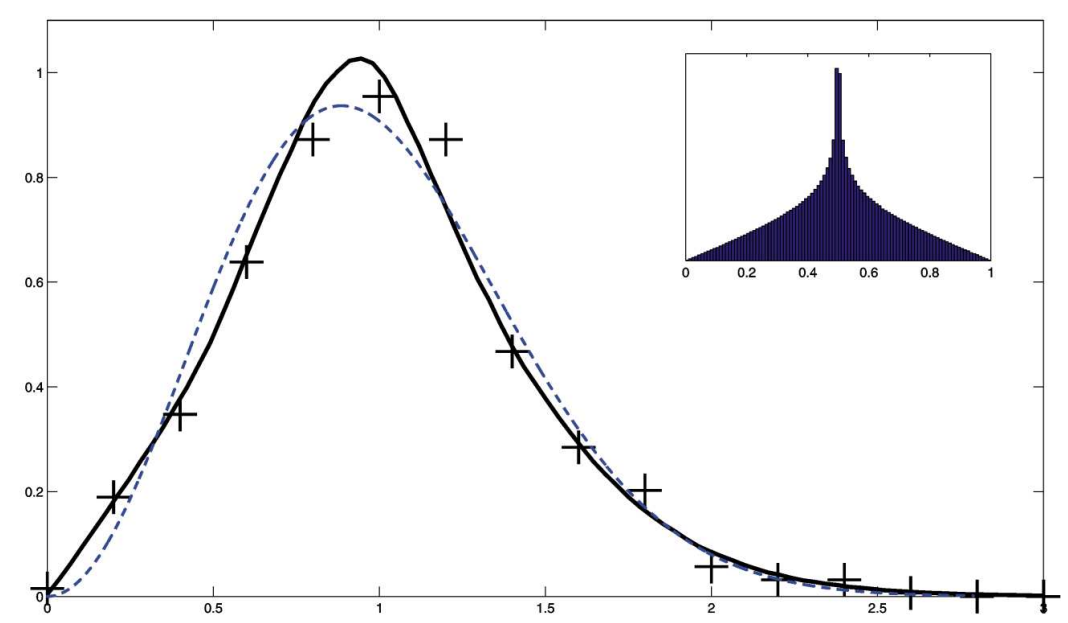

Fig. 3. The measured distance distribution for cars parking on the right side (crosses) is compared with the prediction of GUE (dashed line) and with a solution (8) obtained for $P(a)$ plotted in the inset.

The resulting distribution depends on the choice of the $P(a)$. We display the measured results in Figs. 3 and 4 where the first one shows the result for the right side parking and the second for a left side one. In both cases the results are quite close to the random matrix prediction (one to the GUE and the second to GOE ensemble). But the agreement is not perfect. In both cases the description with the model leads to better results than the random matrix theory. In addition the density $P(a)$ (the figure insets) display clear dependence on the parking maneuver. For the right side parking we used for $a \in(0,0.5)$ : 


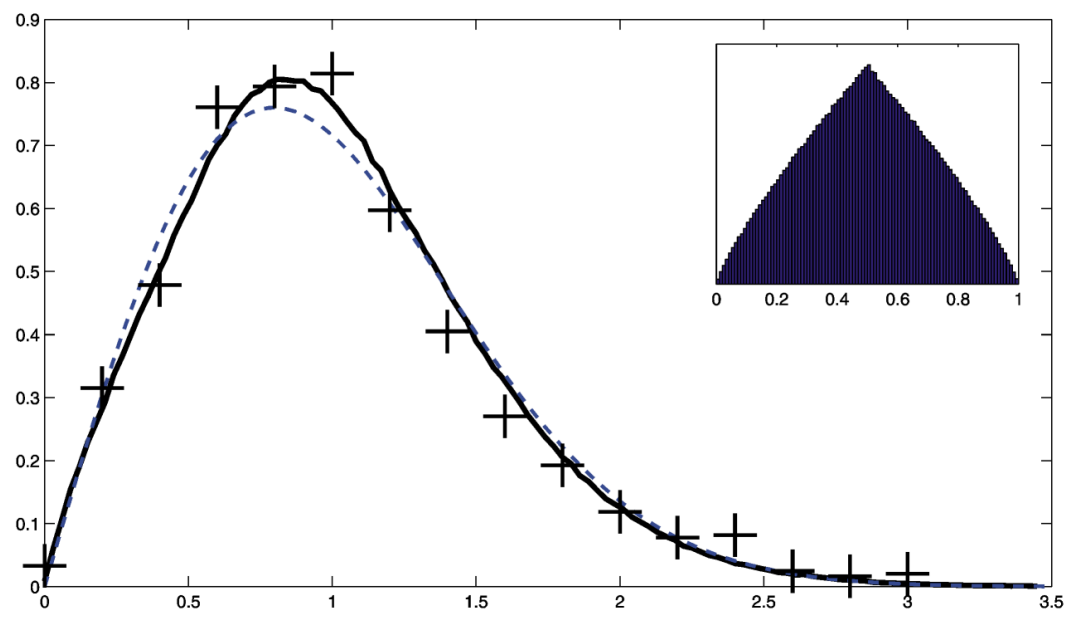

Fig. 4. The measured distance distribution for cars parking on the left side (crosses) is compared with the prediction of GOE (dashed line) and with a solution (8) obtained for $P(a)$ plotted in the inset.

$$
P(a) \approx \frac{a}{(0.5-a)^{\alpha}}
$$

with $\alpha=0.2$. This distribution is biased to $a=0.5$ with a singularity at this point. In the left side case we used a nearly triangular distribution: $P(a) \approx a^{\alpha}$ with $\alpha=0.92$. In both cases $P(a)$ is completed as $P(a)=P(1-a)$ for $a \in(0.5,1)$. Let us notice that — as expected — when the driver parks on the left side the probability he comes closer to the front or back car is higher than in the right side parking case. However the exact profile of $P(a)$ is not a crucial issue since the final result does not depend sensitively on it.

In reality the car lengths are not equal. But this does not represent a serious problem. This situation can be described with Eq. (8) as well. The only change is that now instead of 1 there will be another random variable. Numerical simulation shows that the resulting distribution $\rho(D)$ is not changed when this variable has a symmetric density and reasonably small variance and mean equal to 1 .

\section{Summary}

To summarize: we have shown that car parking can be reasonably well described by a simple model where the distance distribution is a solution of a distributional fixed point equation. The model predictions were compared with data obtained by measuring the distances between cars parked in the center of Hradec Králové. Moreover it has been demonstrated that the distributional fixed point equation leads to solutions that are in astonishing agreement with the distribution of level spacings of the Gaussian orthogonal and unitary random matrix ensembles. 


\section{Acknowledgments}

The research was supported by the Czech Academy of Sciences and Ministry of Education, Youth and Sports within the projects A100480501 and LC06002. The help of the Ph.D. students Michal Musilek and Jan Fator who collected the used car distance data is gratefully acknowledged.

\section{References}

[1] J.W. Evans, Rev. Mod. Phys. 65, 1281 (1993).

[2] A. Cadilhe, N.A.M. Araujo, V. Privman, J. Phys. Condens. Mater 19, 065124 (2007).

[3] A. Renyi, Publ. Math. Inst. Hung. Acad. Sci. 3, 109 (1958).

[4] R. Arnott, E. Inci, J. Urban Econom. 60, 418 (2006).

[5] H.J. Griffionen-Joung, H.J.W. Janssen, D.J. van Amelsfoork, J.J. Langefeld, in: 8th Europ. Conf. on Mobility Management ECOMM 2004, Grand Lyon Communaute Urbaine, 2004, p. 1.

[6] S. Hess, J.W. Polak, Mixed Logit Estimation of Parking Type Choice, Presented at 83rd Annual Meeting of the Transportation Research Board, Washington DC, 2004.

[7] S. Rawal, G.J. Rodgers, Physica A 246, 621 (2005).

[8] N.A.M. Araujo, A. Coadilhe, Phys. Rev. E 73, 051602 (2006).

[9] M.R.D. Orsogna, T. Chou, J. Phys. A 38, 531 (2005).

[10] X.F. Yang, K.M. Knowles, J. Am. Ceram. Soc. 75, 141 (1992).

[11] J.K. Mackenzie, J. Chem. Phys. 37, 723 (1962).

[12] A.Y. Abul-Magd, Physica A 368, 536 (2006).

[13] F.J. Dyson, J. Math. Phys. 3, 140 (1962).

[14] M.L. Mehta, Random Matrices, 2nd ed., Academic, New York 1991.

[15] A. Fader, The gap size distribution of parked cars and the Coulomb gas model, Mathematics REV Paper, 2006, available at http://www.umich.edu/afader.

[16] L. Devroye, R. Neininger, Adv. Appl. Prob. 34, 441 (2002).

[17] D.J. Aldous, A. Bandyopadhyay, Ann. Appl. Prob. 15, 1047 (2005).

[18] M.D. Penrose, A.R. Wade, Adv. Appl. Prob. 36, 691 (2004).

[19] G. Tenenbaum, Introduction to Analytic and Probabilistic Number Theory, Cambridge Univ. Press, Cambridge 1995.

[20] A. Gnedin, S. Kerov, Combinat. Prob. Comput. 10, 213 (2001).

[21] R. Arratia, A.D. Barbour, S. Tavare, Combinat. Prob. Comput. 8, 407 (1999).

[22] Hsien-Kuei Hwang,Tsung-Hsi Tsai, Combinat. Prob. Comput. 11, 353 (2002).

[23] T. Huillet, eprint arXiv:cond-mat/0412166.

[24] R. Aguech, N. Lasmar, H. Mahmoud, J. Appl. Prob. 43, 1 (2006). 